\title{
The Main Treatment of Cancer
}

\author{
Somayeh Zaminpira* and Sorush Niknamian \\ Cellular and Molecular Biology, University of Cambridge, United Kingdom
}

Submission: September 28, 2017; Published: October 16, 2017

*Corresponding author: Somayeh Zaminpira, Ph.D. in Cellular and Molecular Biology, University of Cambridge, United Kingdom, Email: sorushniknamian61@gmail.com

\begin{abstract}
This meta-analysis research has gone through more than 200 studies from 1934 to 2016 to find the differences and similarities in cancer cells, mostly the cause. The most important difference between normal cells and cancer cells is how they respire. Normal cells use the sophisticated process of respiration to efficiently turn any kind of nutrient that is fat, carbohydrate or protein into high amounts of energy in the form of ATP. This process requires oxygen and breaks food down completely into harmless carbon dioxide and water. Cancer cells use a primitive process of fermentation to inefficiently turn either glucose from carbohydrates or the amino acid glutamine from protein into small quantities of energy in the form of ATP. This process does not require oxygen, and only partially breaks down food molecules into lactic acid and ammonia, which are toxic waste products. The most important result is that fatty acids or better told fats cannot be fermented by cells. This research mentions the role of ROS and inflammation in causing mitochondrial damage and answers the most important questions behind cancer cause and mentions some beneficial methods in preventing and treatment of cancer
\end{abstract}

Abbreviations: Cancer; Respiration; Fermentation; ROS; Prevention

\section{Introduction}

\section{Eukaryotic Cell Respiration}

In eukaryotic cells, the respiration process goes through aerobic and anaerobic. The aerobic respiration mainly happens in mitochondrion which requires oxygen for creating Adenosine 3-Phosphate(ATP). Despite consuming protein, carbohydrates and fats as reactants, it is the preferred method of pyruvate breakdown in glycolysis and requires that pyruvate enter the mitochondria in order to be fully oxidized by the Krebs cycle. The products of this process are carbon dioxide and water, but the energy transferred is used to break bonds in ADP as the third phosphate group is added to form ATP (adenosine triphosphate), by substrate-level phosphorylation, NADH and 2FADH2.

$$
\mathrm{C}_{6} \mathrm{H}_{12} \mathrm{O}_{6}(\mathrm{~s})+6 \mathrm{O}_{2}(\mathrm{~g}) \rightarrow 6 \mathrm{CO}_{2}(\mathrm{~g})+6 \mathrm{H}_{2} \mathrm{O}(\mathrm{l})+\text { heat }
$$

The potential of NADH and FADH2 is converted to more ATP through an electron transport chain with oxygen which is the terminal electron acceptor. Most of the ATP produced by aerobic cellular respiration is produced by oxidative phosphorylation. This works by the energy released in the consumption of pyruvate to create a chemi osmotic potential by pumping protons across the cell membrane. This potential is then used to drive ATP synthase and produce ATP from ADP and a phosphate group. By going through the respiration process formula, 38 ATP molecules can be made per oxidised glucose molecule during cellular respiration. Which is, 2 from glycolysis, 2 from the Krebs cycle, and about 34 from the electron transport system. But, this maximum yield is never reached since the losses due to leaky membranes as well as the cost of moving pyruvate and ADP into the mitochondrial matrix, and current estimates range around 29 to 30 ATP per glucose [1].

Aerobic metabolism is almost 15 times more efficient than anaerobic metabolism which yields 2 molecules ATP per 1 molecule glucose. Glycolysis is a metabolic pathway that happens in the cytosol of cells in all living organisms. This pathway can function with or without the presence of oxygen. In humans, aerobic conditions produce pyruvate and anaerobic conditions produce Lactic acid. In aerobic conditions, the process converts one molecule of glucose into two molecules of pyruvic acid, generating energy in the form of two molecules of ATP. Four molecules of ATP per glucose are actually produced; however, two are consumed as part of the preparatory phase. The initial phosphorylation of glucose is required to increase the reactivity in order for the molecule to be cleaved into two pyruvate molecules by the enzyme aldolase. During the pay-off phase of glycolysis, four phosphate groups are transferred to ADP by substrate-level phosphorylation to make four ATP, and two NADH are produced when the pyruvate are oxidized.

Glucose $+2 \mathrm{NAD}++2 \mathrm{Pi}+2 \mathrm{ADP} \rightarrow 2$ pyruvates $+2 \mathrm{NADH}+$ $2 \mathrm{ATP}+2 \mathrm{H}++2 \mathrm{H}_{2} \mathrm{O}+$ heat

Starting with glucose, 1 ATP is used to donate a phosphate to glucose to produce glucose 6-phosphate. Glycogen can be converted into glucose 6-phosphate as well with the help of 
glycogen phosphorylase. During energy metabolism, glucose 6-phosphate becomes fructose 6-phosphate. An additional ATP is used to phosphorylate fructose 6-phosphate into fructose 1,6-disphosphate by the help of phosphofructokinase. Fructose 1,6-diphosphate then splits into two phosphorylated molecules with three carbon chains which later degrades into pyruvate. Glycolysis can be translated as sugar splitting [2].

\section{Oxidative phosphorylation}

In eukaryotes, oxidative phosphorylation occurs in the mitochondrial cristae. It comprises the electron transport chain that establishes a proton gradient (chemi osmotic potential) across the boundary of inner membrane by oxidizing the NADH produced from the Krebs cycle. ATP is synthesized by the ATP synthase enzyme when the chemi osmotic gradient is used to drive the phosphorylation of ADP. The electrons are finally transferred to exogenous oxygen and, with the addition of two protons, water is formed [3].

\section{Fermentation}

Without oxygen, pyruvate pyruvic acid is not metabolized. However, goes through the process of fermentation. The pyruvate is not transported into the mitochondrion, but remaining

in the cytoplasm, where it is converted to waste products that may be removed from the cell. This serves the purpose of oxidizing the electron carriers so that they can perform glycolysis again and removing the excess pyruvate. Fermentation oxidizes NADH to NAD+ so it can be re-used in glycolysis. In the absence of oxygen, fermentation prevents the buildup of NADH in the cytoplasm and provides NAD+ for glycolysis. This waste product varies depending on the organism. In skeletal muscles, the waste product is lactic acid.

Fermentation is less efficient at using the energy from glucose because only 2 ATP are produced per glucose, compared to the 38 ATP per glucose theoretically produced by aerobic respiration. This is because the waste products of fermentation still contain chemical potential energy that can be released by oxidation. The total ATP yield in ethanol or lactic acid fermentation is only 2 molecules coming from glycolysis, because pyruvate is not transferred to the mitochondrion and finally oxidized to the carbon dioxide (CO2), but reduced to ethanol or lactic acid in the cytoplasm [4].

\section{Lactic Acid Fermentation}

Lactic acid fermentation is a metabolic process in which glucose is converted to cellular energy and the metabolite lactate. It is an anaerobic fermentation reaction that occurs in some animal cells, such as muscle cells [5-7]. If oxygen is present in the cell, many organisms will undergo cellular respiration. Hence, facultative anaerobic organisms will both ferment and undergo respiration in the presence of oxygen. Sometimes even when oxygen is present and aerobic metabolism is happening in the mitochondria, if pyruvate is building up faster than it can be metabolized, the fermentation will happen anyway. Lactate dehydrogenase catalyzes the inter conversion of pyruvate and lactate with concomitant inter conversion of NADH and NAD+ [5].

\section{Warburg Effect Explanation}

The Warburg effect may be the result of damage to the mitochondria in cancer, or an adaptation to low-oxygen environments within tumors, or a result of cancer genes shutting down the mitochondria because they are involved in the cell's apoptosis which would kill cancer cells. It may also be an effect associated with cell proliferation. Because glycolysis provides most of the building blocks required for cell proliferation, cancer cells and normal proliferating cells have been proposed to need to activate glycolysis, despite the presence of oxygen, to proliferate [8] evidence attributes some of the high aerobic glycolytic rates to an over expressed form of mitochondriallybound hexokinase [9] responsible for driving the high glycolytic activity. In kidney cancer, this effect could be due to the presence of mutations in the $2 \%$ von Hippel- Lindau tumor suppressor gene up regulating glycolytic enzymes, including the M2 splice isoform of pyruvate kinase [10].

In March 2008, Lewis Cantley and colleagues announced that the $2 \mathrm{M} 2 \% \mathrm{M} \% \mathrm{M}$ tumor $\mathrm{M} 2-\mathrm{PK}$, a form of the pyruvate kinase enzyme, gives rise to the Warburg effect. $2 \mathrm{M} 2 \% \mathrm{M} \% \mathrm{M}$ Tumor M2-PK is produced in all rapidly dividing cells, and is responsible for enabling cancer cells to consume glucose at an accelerated rate; on forcing the cells to switch to pyruvate kinase's alternative form by inhibiting the production of tumor M2-PK, their growth was curbed. The researchers acknowledged the fact that the exact chemistry of glucose metabolism was likely to vary across different forms of cancer; but PKM2 was identified in all of the cancer cells they had tested. This enzyme form is not usually found in healthy tissue, though it is apparently necessary when cells need to multiply quickly, e.g. in healing wounds or hematopoiesis [11,12].

\section{Materials and Methods}

This research has gone through over 200 studies to determine the differences and the similarities between the causes of cancer and review the selected important studies: In 1931, Otto Heinrich Warburg found the prime cause of cancer. In his research, he came to the conclusion that Cancer has countless secondary causes. But, there is only one prime cause. The prime cause of cancer is the replacement of the respiration of oxygen in normal human body cells by a fermentation of sugar [13]. In 2012, Sun and Mito conducted a study. 72 patients aged between 58 to 79 years old were involved. Mitochondria were few in number in the majority of prostate cancer cells for cancers with higher degrees of malignancy. The abnormal ultrastructural features of mitochondria were observed, swelling, low matrix electron density, cristae that were scarce, disordered, broken or absent and mitochondrial vacuolation [14]. In 2004, Ishioka studied ovarian tumors in several women. Which concluded that 
most mitochondria were enlarged and had irregular in shape in all cases [15].

In 2011, Elliott and Barnett conducted a study involving 778 patients with breast carcinoma. In 458 cases, the mitochondria were absent. In 145 patients, the mitochondria were present and in 175 patients mitochondria were present but sparse. This study showed that in cancer cells, the mitochondria may be present but most of them do have irregular and abnormal shape [16]. In 1969, a research conducted by Merkew and Epstein on liver cancer and carcinogenesis. They resulted that in liver cancer cells the mitochondria were unevenly distributed within the malignant neoplastic cells. The structure of these mitochondria was also altered. Many mitochondria were elongated with stratification of their cristae. Within some cells, mitochondria showed signs of degeneration. Within some malignant cells, the mitochondria were densely aggregated and often were closely circumscribed by rough endoplasmic reticulum.

The morphology of some mitochondria resembled that of micro-bodies circumscribed by dilated rough endoplasmic recticulum. Significantly, altered mitochondria were found only within malignant cells. The mitochondrial cristae were elongated, more numerous and rigidly stratified and traversed the entire diameter of an individual mitochondrion. Other altered mitochondria within malignant cells were characterized by decreased numbers and size of the cristae [17]. In 2009, a research done by Morillo Gabriel in human cancer cells in vitro and in vivo. The result was the same as the mentioned researches. The mitochondria in cancer cells, independently of histogenesis, predominantly are seen with lucent-swelling matrix associated with disarrangement and distortion of cristae and partial or total cristolysis and with condensed configuration in minor scale. Mitochondrial changes are associated with mitochondrialDNA mutations, tumoral microenvironment conditions and mitochondrial fusion-fission disequilibrium. Functionally, the structural alterations suppose the presence of hypoxia-tolerant and hypoxia-sensitive cancer cells.

Possibly, hypoxia-tolerantcells are related with mitochondrial condensed appearance and are competent to produce adequate amount of ATP by mitochondrial respiration. Hypoxia-sensitive cells are linked with lucent-swelling and cristolysis mitochondria profile and have an inefficient or null oxidative phosphorylation, which consequently use the glycolytic pathway to generate energy. Additionally, mitochondrial fragmentation is associated with apoptosis; however, alterations in the mitochondrial network are linked with the reduction in sensitivity to apoptosis induces or pro-apoptotic conditions [18].

\section{Discussion}

Cancer cells are very different from normal cells. They grow independently, ignoring the anti-growth signals and death cues that would normally keep healthy cells from getting out of control. Cancer cells create their own blood supply and can divide for a long time. Cancer cells lose many of the physical features of their mother cells. They are often smaller, disfigured or shapeless. Sometimes they fuse with each other or with neighboring cells, creating strange hybrids. The most aggressive types of cancer cells invade local tissues or break loose and travel in the bloodstream to distant parts of the body and metastasize. There are many kinds of mutations have been discovered in cancerous cells, but it is actually very rare to find genetic mutations in healthy cells since they have stable DNA. DNA is the most important molecule in the body so evolution has made sure it is well-protected. The DNA of healthy cells is not fragile. There are even so called caretaker genes that are designed to maintain and repair defects in DNA, because lots of things in the natural environment can injure DNA even things one thinks of as healthy.

Cancer cells have unstable DNA, which mutates easily and is constantly changing. This is why there are so many mutations found in cancer cells. This genomic instability is viewed as a strong suit by scientists who believe in the mutation theory. They think that the tumor cells keep mutating to improve themselves, and that the ones with the cleverest mutations are the ones which survive best and reproduce best that is Darwinism which is based on survival of the fittest. They think of cancerous cells as invincible, as stronger, faster, and smarter than healthy cells. But this isn't true. Most tumor cells are growing faster than most of their healthy neighboring cells, but this is not because they are speedier, it is because they are unregulated.

All the healthy cells around them are capable of growing just as fast, but there are checks and balances in place to prevent them from growing. When necessary, they can grow just as fast, if not faster than tumor cells do. For instance, when the liver is injured and healthy cells need to grow rapidly to replace the injured cells; their growth rate is the same as for liver cancer cells during tumor progression. Fragile DNA is not flexible enough or coordinated enough to respond to challenges. It is, after all, the stability of healthy DNA that allows our cells to adapt to stressful environments. Tumor cells are also more sensitive to heat and starvation [2].

\section{Oncogenic paradox}

A huge variety of things in the world from viruses to radiation, chemicals and oxidation can damage DNA and cause mutations.

I. There are hundreds of thousands of unique mutations associated with tumors. A single colon cancer cell can contain 11,000 mutations. The sheer number and type of mutations found in cancer cells are so serious that they would cause a healthy embryo to spontaneously abort.

II. The transformation of a healthy cell into a cancerous cell malignant transformation happens in the very same specific way every time.

There is not one example of a mutation that causes the same type of cancer every time. Even those mutations most 
strongly associated with certain cancers only cause cancer in certain people. Cancer cells within the very same tumor can have different mutation patterns. Mutated genes thought to be strongly associated with cancer oncogenes, sometimes do promote tumor growth, but sometimes they inhibit tumor growth, and sometimes they do both. If one transplant mutated cancer cell DNA into a healthy cell, the healthy cell almost never becomes cancerous. Only 2 out of 24 experiments were successful in transforming 2, 3, 4 normal cells into cancer cells. This result is against the mutation theory of cancer, which explains why mutation theory of cancer is not right.

2, 3, 4 normal cells into cancer cells. This result is against the mutation theory of cancer, which explains why mutation theory of cancer is not right.

\section{Metabolic Theory of Cancer}

Mitochondria turn the food into energy. They are complex structures living within almost all eukaryotic cells. Inside mitochondria are folded membranes studded with special enzymes, fats and proteins that run elegant chemical reactions. These chemical reactions are what produce ATP. Mitochondria float around in the outer region of the cell which is called the cytoplasm. The cell's chromosomes and DNA live inside the nucleus. Mitochondria have their own DNA which is one of the reasons behind endo symbiotic hypothesis of mitochondrion. Mitochondria are sophisticated power generators that break open the chemical bonds within food molecules to get at the energy inside. Chemical bonds consist of positive charges called protons and negative charges called electrons, which hold onto each other tightly.

Mitochondria wrench the electrons away from the protons, and funnel the electrons through an electron transport chain, creating current. This electrical energy is used to create ATP molecules, each of which includes a very high-energy phosphate bond. Adenosine Tri phosphate is like a chemical battery and our cells can break ATP phosphate bonds apart whenever they need energy. Oxygen waits at the end of the ATP assembly line to catch the cascading electrons, and then binds to them, forming water as a harmless by-product. Since this process requires oxygen and results in a high energy phosphate bond, it is called oxidative phosphorylation, or respiration.

The most important fundamental difference between normal cells and cancer cells is how they make energy. Normal cells use the sophisticated process of respiration to efficiently turn fat, carbohydrate, or protein into high amounts of energy. This process requires oxygen and breaks food down completely into carbon dioxide and water. Cancer cells use a primitive process called fermentation to inefficiently turn either glucose which is primarily from carbohydrates or the amino acid glutamine which is from protein into small amounts of energy. The most important findings from these researches are that fats cannot be fermented. This process does not require oxygen, and only partially breaks down food molecules into lactic acid and ammonia, which are toxic waste products. Normal cells sometimes have to change to fermentation process if they are temporarily experiencing an oxygen shortage.

However, no cell in its right condition would ever choose to use fermentation when there is enough oxygen. It doesn't produce nearly as much energy and creates toxic byproducts. Briefly, fermentation is primitive and wasteful. Cancer cells use fermentation even when there's plenty of oxygen around, which is the explanation of the Warburg Effect, considered the metabolic signature of cancer cells. If a cell turning glucose into lactic acid when there is oxygen available, this would be a cancer cell [19].

Cancer cells cannot rely on respiration system for energy production because of the damaged mitochondria. Respiration cannot run normally unless all of the delicate interior structures inside mitochondria are fully intact. Fermentation also happens inside mitochondria, but the key difference is that fermentation is very simple and does not need the complex inner machinery of the mitochondria. Radiation, Cancer-causing chemicals, Viruses and Chronic inflammation can be main cause mitochondrial damage. One way these things can cause problems for mitochondria is by generating reactive oxygen species (ROS), which damage respiration. ROS is like unstable molecular pin balls, wreaking havoc with molecules around them, causing random damage wherever they strike. It just so happens that some of the genes most strongly linked to oncogenes are those that code for mitochondrial proteins [20]. Mutations in BRCA-1 (breast cancer gene), APC (colon cancer gene), RB (retinoblastoma gene), XP (xeroderma pigmentosum gene) can be found in cancer cells [21].

Some of the viruses most strongly linked to cancer are known to damage respiration are, Kaposi' sarcoma virus, Human papilloma virus(cervical cancer),HIV and Cytomegalovirus [22]. Compared to healthy cells, cancer cells have Fewer mitochondria per cell, Misshapen mitochondria with unnaturally smooth inner surfaces, Reduced activity of critical respiration enzymes such as cytochrome oxidase and ATPase, Smaller amounts of deformed cardiolipin (a crucial mitochondrial fat), Less DNA within their mitochondria and Leaky, uncoordinated electron transport chains that cause some precious energy to be wasted as heat instead of turned into ATP. This abnormal situation is called uncoupling. Faster-growing tumors are actually warmer because of this effect [23].

Malignant cancer cells have substantially lower respiration rates compared to normal cells. In one study of human metastatic rectal cancer, the cancerous cells had respiration rates $70 \%$ lower than the surrounding normal cells [24]. Mitochondria evolved a process called the retrograde response, which helps them deal with temporary stress or damage. It is called a retrograde or backwards response for under normal circumstances; the DNA inside the nucleus calls shots and sends orders out to the mitochondria in the cytoplasm. However, if a 
mitochondrion is damaged, and respiration is endangered, the mitochondrion sends an SOS message to the nucleus saying they need fermentation [25].

It essentially tells the nucleus to activate fermentation genes instead of respiration genes. You can think of fermentation as a clunky backup generator. The retrograde response triggers the following events: A variety of genes spring into action-genes that code for proteins required to run fermentation instead of respiration. Myc, Ras, HIF-1alpha, Akt, and m-Tor. These same genes also happen to be known in the cancer research world as oncogenes which are genes that are associated with increased cancer risk. It is likely that the reason why genes needed to run fermentation are also the same genes associated with cancer is that fermentation and lack of respiration increases cancer risk. While these fermentation oncogenes are revving up, their respiration counterparts are gearing down. Genes like p53; APE1 and SMC4, code for DNA repair proteins and are associated with respiration. These same genes also are known as tumor suppressor genes which are genes that prevent cancer. Turning down the activity of DNA repair proteins is not something that is logical and scientifically beneficial. The retrograde response was designed for temporary emergency use. Cancer cells stay in this mode forever because they have no other choice [26-28].

Being in full throttle fermentation mode with respiration only limping along has the following effects: Reactive oxygen species (ROS) are generated, causing random damage[29]. Ironsulfur complexes are injured. These are needed in the electron transport chain[30]. P-glycoprotein is activated, which pumps toxic drugs out of cells. This can make tumor cells resistant to most chemotherapy [31]. The ability of mitochondria to initiate programmed cell suicide (apoptosis) fails [32]. When something serious goes wrong within a cell, it is the mitochondrion's job to make sure the cell bows out gracefully, for the sake of the organism. This is how cancer cells with all kinds of strange mutations survive; fermentation allows weird cells to live on [33].

Calcium leaks out of mitochondria and into the cytoplasm. Proper calcium flow is critical to normal cell division because the mitotic spindle, which is the structure that helps chromosomes separate properly, is calcium-dependent. Faulty spindles increase the risk of lopsided cell divisions-with one daughter cell getting too many chromosomes and the other daughter cell not getting enough [34].

\section{a) Mitochondrial Relation to Cancer}

b) Fusing tumor cytoplasm (mitochondria) with normal cells (with healthy DNA in their nuclei) and then injecting these hybrid cells into animals produces tumors in $97 \%$ of animals [35].

c) Transplanting normal cytoplasm (mitochondria) into tumor cells (with mutant DNA in their nuclei) reduces cancerous behavior [36]. d) Fusing normal cytoplasm (mitochondria) with tumor nuclei (with mutant DNA inside) reduces the rate and extent of tumor formation [37].

e) If normal cytoplasm (mitochondria) pre-repeated with radiation, it loses its ability to rescue tumor cells from cancerous behavior since radiation damages mitochondria [38].

f) Transferring healthy mitochondria into cells with damaged mitochondria reduces cancerous behavior [39].

The status of the DNA is not what is important. Damaged mitochondria can turn healthy cells into cancerous cells and healthy mitochondria can reverse cancerous behavior in tumor cells. This tells that cancer is not a genetic disease. Cancer is a mitochondrial disease.

\section{Damaged Mitochondria and Cancer}

Billions of years ago, before plants took hold on the planet, earth's atmosphere had very little oxygen and living creatures used fermentation to generate energy. Organisms were very simple, without sophisticated controls to help them decide when to reproduce. They just reproduced as fast as they possibly could [40]. Mitochondria appeared about 1.5 billion years ago, about a billion years after oxygen became available, and probably already had the ability to switch back and forth between fermentation and respiration, depending on how much oxygen was around. Many cells will simply extinct if their mitochondria are damaged, but if the damage is not too sudden or severe, some cells will be able to adapt and survive by switching back to fermentation to make energy [41].

Mitochondrial damage unlocks an ancient toolkit of preexisting adaptations that allow cells to survive in low-oxygen environments. Mitochondria are so good at producing energy that their arrival on the evolutionary scene is thought to be largely responsible for the increase in complexity of living things. Building and supporting elaborate new creatures with specialized organs and capabilities takes a lot of energy. If an organism does not constantly pour energy into a living thing to maintain its form and function, it will gradually succumb to entropy or chaos. For cells, this means regressing which means DNA becomes unstable [42].

Cells lose their unique shapes, become disorganized and start reproducing uncontrollably. Any number of environmental hazards can damage mitochondria. These are the same kinds of things thought typically as damaging DNA and causing cancer. However, the previous lines in this research article proposed that damaged DNA is not the primary cause of cancer. It is the mitochondria that are responsible. Mitochondria take care of cells and DNA. Mitochondrial damage happens first, and then genetic instability follows. Even though there is plenty of oxygen around, damaged mitochondria have no choice but to resort to fermentation, which is primitive and wasteful. Cells cannot stay in shape and under control under these circumstances. They 
may be able to live, but it will not be normal. Cells with damaged mitochondria, only if they survive, are at high risk for becoming cancerous [43].

\section{Cancer and Sugar}

Nearly all tumors depend heavily on glucose for survival, which is how PET scans are able to find many tumors hiding in normal tissues. PET scans follow radioactive glucose as it travels through the bloodstream. Radio-labeled glucose accumulates in tumor tissue more than in the normal tissues surrounding it, and lights up on the scan. There is a strong connection between high blood sugar or hyperglycemia, diabetes, and cancer. It is welldocumented that the growth of brain tumors is more accelerated and prognosis is worse in animals and human with higher blood glucose levels [44].

Hyperglycemia is directly linked with poor prognosis in humans with malignant brain cancer and is connected to the rapid growth of most malignant cancers. High blood glucose raises insulin levels, which stimulates cancer cells to take in and use more glucose [45]. This makes it easier for cancer cells to nourish themselves. Insulin also turns up the activity of the fermentation pathway, and fermentation leads to additional cellular damage. High blood glucose also raises levels of another circulating hormone called IGF-I or Insulin-like Growth Factor I. Cancer cells with receptors on their surfaces for this hormone grow more rapidly. IGF-I turns on a chemical pathway that drives tumor cell growth.

Which is the PI3K-Akt-HIF-1 alpha pathway? This pathway sets the stage for cells to multiply, escape apoptosis, and recruit their own angiogenesis. Angiogenesis is required for tumors to grow beyond 2 millimeters in size. The genes for this growth pathway are also turned up by the fermentation process. More glucose means more fermentation and more insulin and more IGF-I mean more tumor growth. Briefly, cancer is a disease of growth, and insulin is the mother of all growth hormones[46].

Regardless of which type of cancer one may have, what grade or stage it might be, or which mutations or genetic markers it might have, the hallmark of all cancer cells is damaged mitochondria. Cancer is not a collection of unrelated diseases that each need to be treated individually, cancer is one disease which is a mitochondrial disease, and diseased mitochondria prefer glucose and glutamine for fuel. Healthy cells with healthy mitochondria are flexible and can adapt to just about any fuel source, but not cancer cells. In fact, the majority of cells function best when they burn fat for energy. Cancer cells are bad at burning fat, because fat burning requires respiration, which requires healthy mitochondria [47].

\section{Cancer Treatment}

If food is restricted enough to lower blood glucose, then insulin and IGF-1 levels will also be lower, quieting the tumor driving genes and pathways which is described in previous lines. This means that fermentation becomes harder for tumors to recruit new blood vessels, and tumor growth slows. Under low blood glucose levels, glucagon comes in. this is the opposite of insulin hormone. Glucagon stimulates fat burning, which raises ketones and fatty acids in the blood. Ketones and fatty acids are just breakdown products of fats [48].

Ketone bodies and fatty acids cannot be fermented, thus, cancer cells cannot utilize them for fuel. Glucose restriction stresses cancer cells. However, most healthy cells prefer to use fatty acids and ketones for energy. Glucose restriction is good for healthy cells. Glucagon also keeps blood sugar from dropping too low by turning on a process in the liver called gluconeogenesis. This is why humans never need to eat any carbohydrates. Humans are always able to make all the glucose they need out of proteins and fats [49].

The brain cannot burn fatty acids but it can burn ketones, and under low glucose conditions, the brain gradually shifts from burning mostly glucose to burning mostly ketones. The brain may still require a small percentage of glucose to function at its best, but there is always enough glucose in the bloodstream because of glucagon, and most other organs will pass up glucose under these conditions in order to let the brain have first dibs. Cancer cells and healthy cells both have a molecule on their surfaces called GLUT-1 [50].

This glucose transporter ushers glucose out of the bloodstream and into cells. Under low glucose conditions, healthy cells will create more of these transporters and display them on their surfaces so as to optimize their ability to obtain glucose. Cancer cells, which are damaged, and therefore less flexible and adaptable, are not able to do this. In fact, when glucose levels are low, cancer cells are even weaker than usual. Not only can they not raise their GLUT-1 levels, their GLUT-1 levels actually drop. This is one more way that glucose restriction impairs cancer cells. Even though there is always some glucose in the bloodstream because of gluconeogenesis, cancer cells are less able to access it than healthy cells because they are damaged [51].

When ketones are burned for energy instead of glucose, fewer reactive oxygen species (ROS) are generated. These are free radicals that cause oxidative damage. This means that shifting the body from being a carbohydrate-burning machine to becoming a fat-burning machine reduces oxidative damage, and therefore potentially reduces risk for numerous chronic diseases. Diets that raise blood levels of ketones are considered to be neuro protective. That is they protect brain cells from harm. Glucose burning is neurotoxic and burning ketones instead simply restores the natural, healthy level of disease resistance humans inherited from their ancestors [52].

One reason why ketogenic diet is under consideration for the treatment of so many neurological diseases, from autism to Alzheimer's to multiple sclerosis and epilepsy to Parkinson's Disease, is that the transition from glucose burning to ketone burning is powerfully anti-inflammatory. There is no drug 
therapy can target as many pro-inflammatory mechanisms in the microenvironment as can dietary energy restriction. Real progress in tumor management will be achieved once patients and the oncology community come to recognize this fact. In fact, it is inflammation which damages mitochondria and respiration, and thus, inflammation may be the true cause of cancer [53].

\section{Starving Cancer Cells to Death}

Food restriction reduces the incidence of both inherited and acquired cancers in laboratory animals [1] .Most cancer cells grow best when they have access to a combination of glucose and the amino acid glutamine. But, there are some types of cancer cells which do just fine without any glucose as a food source, because they are especially good at burning glutamine. This is why both glucose from dietary carbohydrates and glutamine from dietary protein, need to be restricted in order to best target cancer cells. Therefore, a low-calorie ketogenic diet consisting of $80 \%$ fat, with the rest $20 \%$ being made up of protein plus carbohydrate maybe the best way to starve cancer cells. This diet forces normal cells to burn fat for energy [54]. It contains enough protein for normal cells to function properly. Excess protein means excess amino acids and glutamine. The ketogenic diet does not have to contain any carbohydrate, However, it is not harmful if contains significant amounts of carbohydrate, as long as calories are kept low. Blood glucose levels respond more to calorie intake than to carbohydrate intake [55].

The goal of this specific diet is to shift the body from burning mostly glucose to burning mostly ketones. Fat molecules get broken down into 3 fatty acid chains plus one molecule of glycerol. The fatty acids can be turned into ketones, and the glycerol backbone can be turned into glucose. This is why even eating too much fat can raise blood sugar a little bit in some people. Carbohydrates are best at causing high blood sugar $[55,56]$. Proteins can raise blood sugar because some amino acids can be turned into glucose. Dietary fat is least likely to raise blood sugar, but it is not impossible, specifically, if one is eating more calories than he needs. The idea behind ketogenic diet is to restrict carbohydrate and protein so much that fat from the diet is broken down into ketones instead of being stored as fat, which are burned by healthy cells for energy [54-59].

\section{Summary and Conclusion}

\section{Standard Therapies}

a) Conventional treatments can help in the short-term but can cause problems in the long-term.

b) Chemotherapy is toxic to healthy cells and can breed resistance among cancer cells, increasing the risk of more aggressive cancers if relapse occurs.

c) Radiation turns up the activity of the tumor growth pathway via PI3K-Akt-HIF, which promotes not only tumor growth, but also recruitment of angiogenesis and drug resistance. d) Radiation increases fusion activity between cells, which means that normal and healthy cells can merge into hybrid cells and become more aggressive

e) Radiation directly damages mitochondria, which increases risk for cancer in the future

f) Both radiation and immune suppression therapy can increase the incidence of metastatic cancers.

g) Steroids such as dexamethasone, often used to reduce inflammation, raise blood sugar levels, feeding tumor cells and enhancing their survival.

\subsection{Dietary Therapies}

a) Dietary energy restriction triggers cancer cell death via apoptosis which is a natural, non-inflammatory process that happens within cells, causing no collateral damage. Conventional treatments kill cancer cells via necrosis, an inflammatory process that happens from the outside and is locally destructive. Tumor cells that are being fed glucose/ glutamine are resistant to apoptosis, but under ketogenic conditions, they become better able to undergo apoptosis again.

b) DER and chemotherapy can both cause weight loss. However, the weight loss associated with DER is healthy and does not weaken patients, whereas chemotherapy-induced weight loss is unhealthy and weakens patients.

\subsection{Complementary Strategies}

A. Anti-glycolytic drugs that reduce the activity of the glycolysis fermentation pathway, which is the primary energy pathway for most cancer cells.

B. CR-mimetic drugs that mimic the effects of calorie restriction by lowering glucose levels. These drugs should not be used without diet, since they lower glucose without raising ketones. Without ketones, healthy cells could die of energy failure.

C. Hyperbaric oxygen Therapy is beneficial. Excess oxygen reduces the activity of an enzyme called hexokinase II, which grabs onto glucose after it enters cells and traps it inside so it can be burned for energy.

\section{References}

1. Reece, Urry, Cain, Wasserman, Minorsky, et al. (2010) Campbell Biology ( $9^{\text {th }}$ edn). Pearson Education, Inc. p. 168.

2. Porter R, Brand M (1995) Mitochondrial proton conductance and $\mathrm{H}+/ \mathrm{O}$ ratio are independent of electron transport rate in isolated hepatocytes. The Biochemical Journal 310(2): 379-382.

3. Rich PR (2003) The molecular machinery of Keilin's respiratory chain. Biochemical Society Transactions 31( 6): 1095-1105.

4. Stryer, Lubert (1995) Biochemistry New York - Basingstoke: W. H. Freeman and Company.

5. Fermented Fruits and Vegetables - A Global SO Perspective. United Nations FAO. (1998). 
6. Ohio State University (1998) Glycolysis and Fermentation. Retrieved 2010-01-12.

7. Campbell, Neil, Reece, Jane (2005) Biology, ( $7^{\text {th }}$ edn). Benjamin Cummings.

8. Lopez Lazaro M (April 2008) The warburg effect: why and how do cancer cells activate glycolysis in the presence of oxygen? Anti-Cancer Agents in Medicinal Chemistry 8(3): 305-312.

9. Bustamante E, Pedersen PL (1977) High aerobic glycolysis of rat hepatoma cells in culture: role of mitochondrial hexokinase. Proceedings of the National Academy of Sciences of the United States of America 74(9): 3735-3739.

10. Unwin RD, Craven RA, Harnden P, Hanrahan S, Totty N, et al. (2003) Proteomic changes in renal cancer and co-ordinate demonstration of both the glycolytic and mitochondrial aspects of the Warburg effect. Proteomics 3(8): 1620-1632.

11. Christofk HR, Vander Heiden MG, Harris MH, Ramanathan A, Gerszten $\mathrm{RE}$, et al. (March 2008) The M2 splice isoform of pyruvate kinase is important for cancer metabolism and tumour growth. Nature 452(7184): 230-233.

12. Pedersen PL (2007) Warburg, me and Hexokinase 2: Multiple discoveries of key molecular events underlying one of cancers' most common phenotypes, the "Warburg Effect", i.e., elevated glycolysis in the presence of oxygen. Journal of Bioenergetics and Biomembranes 39(3): 211-222.

13. Brand RA (2010) Biographical Sketch: Otto Heinrich Warburg, PhD, MD. Clinical Orthopaedics and Related Research 468(11): 2831-2832.

14. X SUN, N K LIAO, J J YU (2012) Prognostic Value of a Mitochondrial Functional Score in Prostate Cancer. The Journal of International Medical Research 40: 371 - 376.

15. Shin ichi Ishioka, Satoru Sagae, Eiki Ito, Ryuichi Kudo (2004) Ultrastructural study of benign, low-malignant potential (LMP), and malignant ovarian tumors. The Clinical Electron Microscopy Society of Japan 2004, Med Electron Microscopy 37: 37-44.

16. Elliott, Barnett (2011) Ultrastructural Observation of Mitochondria in Human Breast Carcinoma Cells Microsc Microanal Microscopy Society of America 2011.

17. Merkew LP, Epstein SM, Farber E, Pardo M, Bartus B (1969) Cellular analysis of liver carcinogenesis 3. Comparison of the ultra structure of hyper plastic liver nodules and hepato cellular carcinomas induced in rat liver by 2-fluorenyl acetamide, Journal of the National Cancer Institute, Article 43(1): 33-63.

18. Arismendi Morillo Gabriel (2009) Electron microscopy morphology of the mitochondrial network in human cancer, The International Journal of Biochemistry \& Cell Biology 41(10):2062-2068

19. Thomas N Seyfried, Roberto E Flores, Angela M, Dominic P D Agostino (2013) Cancer as a metabolic disease: implications for novel therapeutics. Carcinogenesis 35(3): 515-527.

20. Parkin DM, Boyd L, Walker LC (6 December 2011) The fraction of cancer attributable to lifestyle and environmental factors in the UK in 2010. British Journal of Cancer 105(Suppl 2): S77-81.

21. Biffi A, Montini E, Lorioli L, Cesani M, Fumagalli F, et al. (2013) Lentiviral Hematopoietic Stem Cell Gene Therapy Benefits Metachromatic Leukodystrophy. Science 341(6148): 1233158.

22. Pezzoli D, Chiesa R, De Nardo L, Candiani G (2012) We still have a long way to go to effectively deliver genes! Journal of Applied Biomaterials \& Functional Materials 10(2): 82-91.

23. Aiuti A, Biasco L, Scaramuzza S, Ferrua F, Cicalese MP (2013) Lentiviral Hematopoietic Stem Cell Gene Therapy in Patients with WiskottAldrich Syndrome. Science 341(6148): 1233151.
24. Whyte DA, Al-Hammadi S, Balhaj G, Brown OM, Penefsky HS, et al. (2010) Cannabinoids inhibit cellular respiration of human oral cancer cells. Pharmacology 85(6): 328-335.

25. Tuuli Kaambre, Vladimir Chekulayev, Igor Shevchuk, Kersti Tepp, Natalja Timohhina (2013) Metabolic control analysis of respiration in human cancer tissue. Front Physiol 4: 151.

26. Gallagher, James (2015) Gene therapy: Tame HIV used to cure disease BBC News, Health.

27. Malech HL, Ochs HD (2015) An Emerging Era of Clinical Benefit from Gene Therapy. JAMA 313(15): 1522.

28. Lee, Tim WR, Southern KW (2013) Topical cystic fibrosis transmembrane conductance regulator gene replacement for cystic fibrosis-related lung disease. Cochrane Database Syst Rev 11(11): CD005599.

29. MacLaren RE, Groppe M, Barnard AR, Cottriall CL, Tolmachova T, et al. (2014) Retinal gene therapy in patients with choroideremia: Initial findings from a phase $1 / 2$ clinical trial. The Lancet 383(9923): 11291137.

30. Tebas P, Stein D, Tang WW, Frank I, Wang SQ et al. (2014) Gene Editing of CCR5 in Autologous CD4 T Cells of Persons Infected with HIV. New England Journal of Medicine 370(10): 901-910.

31. Collection and Storage of Umbilical Cord Stem Cells for Treatment of Sickle Cell Disease; Clinical Trials.gov Identifier: NCT00012545 ClinicalTrials.gov, U.S. National Institutes of Health, USA (2015).

32. Gardner MR, Kattenhorn LM, Kondur HR, von Schaewen M, Dorfman T, et al. (2015) AAV-expressed eCD4-Ig provides durable protection from multiple SHIV challenges. Nature 519(7541): 87-91.

33. Wade, Nicholas (2015) Scientists Seek Ban on Method of Editing the Human Genome. New York Times. Retrieved 20 March 201

34. Rogers S, New Scientist 1970 p. 194.

35. Matthew G Vander Heiden, Lewis C Cantley, Craig B Thompson (2009) Understanding the Warburg Effect: The Metabolic Requirements of Cell Proliferation Science 324(5930): 1029-1033.

36. Deberardinis RJ, Lum JJ, Hatzivassiliou G, Thompson CB Cell (2008) Metab 7:11.

37. Weinhouse SZ, Krebsforsch Klin Onkol (1976) Cancer Res Clin Oncol $87: 115$.

38. Shaw RJ (2004) Proc Natl Acad Sci USA 101: 3329.

39. Christofk HR, Vander Heiden MG, Wu N, Asara JM, Cantley LC (2008) Pyruvate kinase M2 is a phosphotyrosine-binding protein. Nature 452(7184): 181-1886.

40. Wolf U (1974) Theodor Boveri and his book, on the problem of the origin of malignant.

41. Cairns J (1981) The origin of human cancers. Nature 289: 353-357.

42. Loeb LA (2001) A mutator phenotype in cancer. Cancer Res 61: 32303239.

43. Wagner RP (1999) Anecdotal, historical and critical commentaries on genetics. Rudolph Virchow and the genetic basis of somatic ecology Genetics 151: 917-920.

44. Howell AN, Sager R (1978) Tumorigenicity and its suppression in cybrids of mouse and Chinese hamster cell lines. Proc Natl Acad Sci USA 75: 2358-2362.

45. Jonasson J (1977) The analysis of malignancy by cell fusion. VIII. Evidence for the intervention of an extra-chromosomal element. J Cell Sci 24: 255-263.

46. Seyfried TN (2012) Mitochondria: the ultimate tumor suppressor. In Cancer As A Metabolic Disease: On the Origin, Management, and 
Prevention of Cancer John Wiley \& Sons, Hoboken, New Jersy, USA, pp. 195-205.

47. Kaipparettu ba, Yewei Maa, Jun P Park, Tin Lap Lee, Y Zhang, et al. (2013) Crosstalk from non-cancerous mitochondria can inhibit tumor properties of metastatic cells by suppressing oncogenic pathways.

48. Israel BA, Schaeffer WI (1988) Cytoplasmic mediation of malignancy. In Vitro Cell Dev Biol 24: 487-490.

49. Nelson DL (2008) Lehninger Principles of Biochemistry. W H Freeman, New York, USA.

50. Warburg O (1969) Revidsed Lindau lectures: the prime cause of cancer and prevention - Parts 1 \& 2. In: Burk D (Edn.), Meeting of the Nobel-Laureates. K.Triltsch, Lindau, Lake Constance, Germany.

51. Stellingwerff T, Leblanc PJ, Hollidge MG, Heigenhauser GJ, Spriet LL (2006) Hyperoxia decreases muscle glycogenolysis, lactate production, and lactate efflux during steady-state exercise. Am J Physiol Endocrinol Metab 290(6): E1180-E1190.

52. Koppenol WH, Bounds PL, Dang CV (2011) Otto Warburg's contributions to current concepts of cancer metabolism. Nat Rev Cancer 11(8): 325-337.
53. Chevrollier A, Loiseau D, Gautier F, Malthiery M, Stepein G (2005) ANT2 expression under hypoxic conditions produces opposite cellcycle behavior in 143B and HepG2 cancer cells. Mol Carcinog 42(1): $1-8$

54. Donnelly M, Scheffler IE (1976) Energy metabolism in respirationdeficient and wild type Chinese hamster fibroblasts in culture. In: German J (Edn.), Chromosomes and Cancer, John Wiley \& Sons, New York, USA, p. 1-20.

55. Valter D Longo, Luigi Fontana (2010) Calorie restriction and cancer prevention: metabolic and molecular mechanisms, Trends Pharmacol Sci 31(2): 89-98.

56. Cancer Fact sheet $\mathrm{N}^{\circ} 297$. World Health Organization (2014).

57. Defining Cancer. National Cancer Institute (2014).

58. Cancer - Signs and symptoms. NHS Choices (2014).

59. Watanabe M, Suzuki K (1989) Heat sensitivity of human cancer cells and abnormal expression of heat shock protein 70 . Gan No Rinsho 35(13): 1512-1516. 